\title{
A UTILIZAÇÃO DO CONTROLE ESTATÍSTICO DO PROCESSO (CEP) PARA ANÁLI- SES DOS PARÂMETROS DE COMPOSIÇÃO DO LEITE CRU REFRIGERADO
}

\author{
Bruno Rafael de Oliveira de Souza (EPA/UNESPAR-FECILCAM, Brasil) bruno_epa@yahoo.com.br \\ Camila Pieczarcka Bucioli (EPA/UNESPAR-FECILCAM, Brasil) camila.p.bucioli@gmail.com \\ Lorena Aguilar Seixas (EPA/UNESPAR-FECILCAM, Brasil) lorenaseixas91@gmail.com \\ Rony Peterson da Rocha (GEPPGE, DEP/UNESPAR-FECILCAM, Brasil) petersonccbpr@hotmail.com
}

\begin{abstract}
Resumo: Desde os primórdios o leite vem sendo cada vez mais indispensável na alimentação humana. Com isso, a preocupação com a segurança alimentar e com a qualidade do leite vem sendo cada vez mais observada e controlada a fim de se obter alimento seguro para o consumidor. As análises sobre a composição do leite se tornaram rotina no dia-a-dia dos laticínios. Sendo assim, o presente artigo tem por objetivo realizar uma análise do leite recebido por um laticínio na cidade de Iretama no Paraná, verificando, através do Controle Estatístico do Processo (CEP) se o leite cru refrigerado recebido pela indústria está respeitando os parâmetros estabelecidos por lei. Para realizar esse estudo, foram levantados dados de um mês de trabalho sobre as análises realizadas no leite cru refrigerado no instante de sua coleta para o transporte até a indústria. Foi empregado as cartas de controle do processo para averiguar se os índices das análises de teor de gordura, proteína, Alizarol, acidez e extrato de seco desengordurado (ESD) estavam em conformidade com a normativa 62 . Os resultados mostram que as análises de ESD e de proteína estão dentro dos limites de controle e as demais com alguma alteração no processo. Sugere-se, que para um estudo mais aprofundado dos parâmetros avaliados, uma avaliação com no mínimo seis meses de dados, para um estudo mais concreto ao longo do processo.
\end{abstract}

Palavras-chave: Leite. Análises físico-químicas. Controle estatístico da qualidade (CEQ). Controle Estatístico do Processo (CEP).

\section{Introdução}

O leite in natura, segundo Ferreira (2008) é um produto obtido higienicamente, através de ordenha completa e ininterrupta de vacas sadias, devendo ser resfriado imediatamente após sua obtenção, podendo ser proveniente de várias fêmeas e de uma ou mais ordenhas.
O Brasil é o $4^{\circ}$ produtor mundial de leite com 32 bilhões de litros, cerca de 5,3\% da produção mundial, com uma produtividade de 1.3811/ano. A produção cresceu $4,4 \%$ ao ano de 2000 a 2011. Há registros da produção de leite em 555 microrregiões geográficas das 558 existentes no Brasil (ALVIM, 2013). 


\section{ReLAInEP}

Para Silva (1997), “desde o nascimento do ser humano, o leite apresenta-se quase indissociável de sua alimentação. A melhoria na qualidade de vida do ser humano é ressaltada pela vital necessidade de se ter alimentos saudáveis". Por isso, é de fundamental importância realizar o controle higiênico-sanitário do leite buscando a qualidade do produto e a garantia de um alimento seguro para o consumidor. Por isso é necessário realizar análises do leite in natura diariamente e mensalmente para garantir a qualidade do mesmo.

Este artigo tem por objetivo verificar, através do controle estatístico do processo, se o leite que chega à indústria está seguindo as normas imposta pelo órgão responsável para assegurar a qualidade do leite realizando um estudo com as análises, das características que compõem o leite. Análises estas, feitas no momento da coleta do leite cru refrigerado na propriedade rural e transportado a granel até a recepção na indústria. Para tal, utilizaremos o CEP empregando as cartas de controle como ferramenta da qualidade para realizar as avaliações das características que compõem o leite. O presente artigo se enquadra, dentro da Engenharia de Produção, na área de Engenharia de Qualidade (EQ), já que um dos objetivos é realizar a verificação se o leite que chega à indústria está dentro dos padrões estabelecidos. E dentro da EQ se enquadra na subárea de Gestão de Sistemas da Qualidade.

$\mathrm{O}$ artigo está dividido em seis seções, sendo elas: introdução, fundamentação teórica, metodologia, revisão da literatura, resultados e discussões e conclusões.

\section{Fundamentação teórica}

De acordo com a Normativa $n^{\circ} 62$ (IN62), de 29 de dezembro de 2011, aprovada pelo Ministério da Agricultura e Meio Ambiente (MAPA). A coleta do leite cru refrigerado deve ser realizada com caminhões isotérmicos construídos inteiramente de aço inoxidável. Através de mangote flexível e bomba sanitária, acionada pela energia elétrica da propriedade rural, pelo sistema de transmissão ou caixa de câmbio do próprio caminhão, diretamente do tanque de refrigeração por expansão direta ou dos latões contidos nos refrigeradores de imersão (MAPA, 2002).

O funcionário encarregado da coleta deve receber treinamento básico sobre higiene, análises preliminares do produto e coleta de amostras, podendo ser o próprio motorista do carro-tanque. Deve estar devidamente uniformizado durante a coleta. A ele cabe rejeitar o leite que não atender às exigências, o qual deve permanecer na propriedade (MAPA, 2002).

O leite recolhido da propriedade rural deve apresentar aspecto líquido branco opalescente homogêneo, com sabor e odor característicos, isento de sabores e odores estranhos. Não devem conter neutralizantes de acidez e reconstituintes de densidade, muito menos resíduos de antibióticos e de outros agentes inibidores do crescimento microbiano. O leite deve seguir os requisitos físicos, químicos, microbiológicos, de contagem de células somáticas e de resíduos químicos. São vários os parâmetros analisados diariamente na indústria, porém neste trabalho 


\section{ReLAInEPY}

serão analisados somente os teores de gordura, proteína, acidez, Alizarol e extrato seco desengordurado (ESD), seguindo a regulamentação da IN 62, conforme relacionados na Tabela 1 (LEITE, 2013).

A seguir serão apresentados alguns estudos a realizados e que tratam do mesmo tem em questão. Miguel et al. (2010), identificou em seu estudo que os padrões analisados estavam dentro dos exigidos pela legislação brasileira, mas ficou constatado que outros estudos devem ser realizados para a melhor avaliação de outros aspectos do leite.

Franco et al. (2011), nos apresenta um estudo realizado com o leite cru, pasteurizado e longa vida afim de comparar as análises de acidez, fosfatase alcalina e peroxidase com a normativa $\mathrm{n}^{\mathrm{o}} 51$ de 18/09/2002, comprovando que as amostras estudadas estavam de acordo com a exigências impostas.

No trabalho abordado por Brito (2013), fica evidente a importância da qualidade do leite para a proteção da saúde humana. Enfoca também nas análises realizadas na indústria e a periodicidade com que elas devem ser realizadas. Brito (2013) ainda conclui que são vários os fatores que levam a perda de qualidade do leite, que podem ser as condições precárias desde o manejo, ordenha até o transporte da matéria-prima à indústria.

Assim, nota-se que todos os trabalhos pesquisados focam na busca da verificação da qualidade do leite em relação as característica de composição do mesmo. Mas nenhum utilizou as cartas de controle para a avaliação desses parâmetros. Por tanto, o presente artigo pode ser considerado inovador, por estar utilizando uma ferramenta da qualidade para a verificação da conformidade do leite recebido pela indústria com a IN 62.

TABELA 1: ÍNDICES DE COMPOSIÇÃO MÍNIMA DO LEITE CRU REFRIGERADO ENTREGUE NA INDÚSTRIA

\section{COMPOSIÇÃO MÍNIMA DO LEITE CRU REFRIGERADO}

\begin{tabular}{ccccc}
\hline Gordura (\%) & Proteína (\%) & Acidez $^{\circ} \mathrm{D}$ & Alizarol $\%$ & Extrato Seco Desengordurado \% (ESD) \\
\hline 3 a 6 & 2,9 a 4 & 13 a 17 & Mín 72 & 8,4 \\
\hline \multicolumn{5}{c}{ Fonte: Durr (2012) }
\end{tabular}

Os componentes do leite, com exceção da água, constituem os sólidos totais e são responsáveis pelo seu valor nutricional. O teor de sólidos determina o valor industrial do leite, pois quanto mais gordura e proteína, maior o rendimento que a indústria terá ao fabricar os derivados lácteos (DURR, 2012).
Segundo Leão et al. (2012) "a análise da composição físico-química do leite é importante, pois também é considerada, junto às analises microbiológicas, parâmetros de qualidade do leite, possibilitando estabelecer, dessa forma um critério de pagamento ao produtor". 
Assegurar a qualidade de um produto ou serviço, não é apenas realizar a inspeção final do mesmo, deve-se incentivar o controle de cada passo no processo de produção evitando que itens defeituosos continuem no processo gerando custos de má qualidade. Para que esse controle seja eficiente usamos o CEP - controle estatístico do processo (BONDUELLE, 2013). Bonduelle (2013) define o CEP como sendo:

Um método preventivo de se comparar continuamente os resultados de um processo com um padrão, identificando, a partir de dados estatísticos, as tendências para variações significativas, eliminando ou controlando estas variações com o objetivo de reduzi-las cada vez mais. Ou seja, é o conjunto de técnicas utilizadas para o controle da qualidade do produto durante cada etapa de fabricação (BONDUELLE, 2013, p. 3).

São várias as ferramentas do CEP para avaliação do processo. No presente trabalho, para a avaliação dos dados coletados, foram utilizadas cartas de controle por variáveis. Segundo Bonduelle (2013), cartas de controle são gráficos de análise e ajuste da variação de um processo em função do tempo, através de duas características básicas: sua centralização e sua dispersão.

O tipo mais comumente usado de gráfico de controle empregado para controlar variáveis é o gráfico X-R. De fato, eles são dois gráficos em um. Um gráfico é usado para controlar a média da amostra, $(\mathrm{X})$. O outro é usado para controlar a variação dentro da amostra pela medida da faixa
(R). A faixa é usada porque é mais simples de ser calculada do que o desvio-padrão da amostra (MELO, 2004, p. 27).

As cartas de controle por variáveis foram escolhidas para uma análise e ajuste da variação do processo em função do tempo, através das características de centralização, observada através da média, e a dispersão, através da amplitude dos dados.

Para a realização do processo, utilizamos os dados apresentados na Tabela 2 na seção de resultados e discussão. Escolhidas as cartas de controle X-R, por permitirem um estudo com uma amostragem pequena.

Para o cálculo dos limites de controle, calculase inicialmente a média e a amplitude da amostra. A média da amostra é calculada com a Equação (1) a seguir.

$$
\mathrm{X}_{\mathrm{m}}=\frac{\sum_{\mathrm{i}=1}^{\mathrm{n}} \mathrm{x}_{\mathrm{i}}}{\mathrm{n}}
$$

onde, $\mathrm{X}_{\mathrm{m}}$ é o valor médio, $\mathrm{n}$ é o número de elementos da amostra e x o valor de cada elemento variando de $1 \mathrm{a}$.

A amplitude da amostra é dada segundo a Equação (2).

$$
R=x_{\text {máx }}-x_{\text {min }}
$$

onde, $R$ é a amplitude, $x_{\text {máx }}$ é o valor máximo encontrado na amostra e $x_{\min } \mathrm{o}$ valor mínimo.

No presente artigo as média e amplitude foram calculadas considerando o intervalo de dois dias. Em seguida, com a média e a amplitude calculada, são definidos os limites superior e 


\section{ReLAlnEP}

inferior de controle da carta $\mathrm{X}$, através das Equações (3) e (4), respectivamente:

$$
L S C X=X_{m}+A 2 \cdot R
$$

com $L S C X$ sendo o limite superior de controle da carta $\mathrm{X}, X_{m}$ o valor médio da amostra, $A 2$ um valor tabelado de acordo com o tamanho de subgrupo e $R$ a média das amplitudes.

$$
L I C X=X_{m}-A 2 \cdot R
$$

com LSCX sendo o limite superior de controle da carta $\mathrm{X}, X_{m}$ o valor médio da amostra, $A 2$ um valor tabelado de acordo com o tamanho de subgrupo e $R$ a média das amplitudes.

Para o cálculo dos limites superiores e inferiores de controle da carta R, são usadas as Equações (5) e (6) respectivamente:

$$
L S C R=R \cdot D 4
$$

onde, $L S C R$ é o limite superior de controle da carta $\mathrm{R}, R$ é a média da amplitude e $D 4$ um valor tabelado de acordo com tamanho de subgrupo.

$$
L I C R=R \cdot D 3
$$

com LICR é o limite inferior de controle da carta $\mathrm{R}, R$ é a média da amplitude e $D 3$ um valor tabelado de acordo com tamanho de subgrupo.

\section{Metodologia}

O artigo em questão foi desenvolvido em um laticínio na região centro ocidental do Paraná. Como são realizadas análises diariamente na indústria, foi levantado dados históricos de análises das características do leite cru refrige- rado no momento da coleta do leite na propriedade rural, considerando análises do dia 1 a 31 de julho de 2013.

Os parâmetros físico-químicos analisados foram a Alizarol, acidez, teor de gordura, de proteína, e ESD. Para o tratamento dos dados foi utilizados o CEP empregando as cartas de controle por variáveis $\mathrm{X}$ e $\mathrm{R}$, para avaliar a conformidade do processo.

O teste de Alizarol permite verificar o perfil de acidez do leite, auxiliando na diferenciação entre o desequilíbrio salino e a acidez excessiva. Permite-nos verificar se o leite está ácido ou alcalino, pela presença do indicador de alizarina. Com essa análise, temos a certificação de segurança se o leite poderá ou não ser pasteurizado (aquecido), já que o leite ácido tende a coalhar quando é submetido ao calor (RODRIGUES, 2005).

A acidez aparente do leite é aquela que ele apresenta quando ainda não houve desenvolvimento da verdadeira acidez, fruto da fermentação da lactose e formação de ácido lático por microrganismos. A acidez é devida à presença de caseína, fosfatos, albumina, dióxido de carbono e Citatos, podendo variar de 13 a $17^{\circ} \mathrm{D}$. Eventualmente essa faixa pode ser mais ampla, dependendo da individualidade e da raça da vaca (RODRIGUES, 2005).

Como em certos países, o pagamento do leite ainda é feito pelo conteúdo em gordura, é muito importante realizar essa análise. Segundo Rodrigues (2005), o extrato seco desengordurado corresponde a todos os componentes do leite, excluindo a gordura e a água, refere-se a parte sólida do leite excluindo a gordura. 


\section{ReLAlnEP}

Para a avaliação do processo foram utilizada cartas de controle por variáveis. As cartas de controle foram empregadas utilizando o software Minitab 15 na versão em espanhol. O software utilizou uma média para o tratamento dos dados e desenvolvimento das cartas de controle, considerando dois dias de amostras como um dia apenas, reduzindo assim o período amostral para 15 dias, realizando o mesmo procedimento para a amplitude das variáveis.

Para o desenvolvimento deste trabalho foram realizadas pesquisas sobre assuntos relacionados ao tema abordado em sites de eventos e encontros que tratem do mesmo e de órgão públicos para a investigação da legislação vigente para a regulamentação dos parâmetros analisados em questão. Como palavras-chave, foram escolhidas palavras de fácil associação com o problema em questão e ao mesmo tempo fosse abrangente ao tema, mas de fácil associação com o artigo proposto, buscando expressões relevantes e coerentes com o conteúdo aqui tratado. As palavras escolhidas foram: leite, análises físico-químicas, CEQ e CEP.

Para o tipo de pesquisa pode ser caracterizada quanto aos objetivos como descritiva, quanto à forma de abordar o problema como qualitativa e quanto à estratégia de pesquisa foi utilizado o estudo de caso. A revisão da literatura foi baseada em artigos científicos que tratam sobre a análise do leite, na coleta e transporte a granel na propriedade e em sua recepção na indústria.

\section{Resultados}

Com o objetivo de averiguar se o processo está em conformidade, foram aplicadas as cartas de controle nos índices de alizarol, acidez, teor de gordura, proteína e extrato seco desengordurado (ESD). A Tabela 2 nos apresenta os dados coletados do dia 1 de julho de 2013 ao dia 31 de julho de 2013 no momento da coleta do leite para o transporte até a indústria e a partir desta Tabela 2, elaborado os gráficos de controle.

A análise desses dados foi realizada com base na teoria do CEP e com na base na IN 62, que estabelece padrão para esses parâmetros, conforme apresentado na Tabela 1.

TABELA 2: DADOS COLETADOS NO MÊ DE JULHO DE 2013

\begin{tabular}{c|ccccc}
\hline Dia/07/13 & Alizarol $^{\mathbf{}} \mathbf{G L}$ & Acidez $^{\mathbf{} D}$ & Gordura \% & ESD \% & Proteína \% \\
\hline 1 & 73,00 & 15,58 & 4,12 & 8,41 & 3,06 \\
2 & 61,32 & 16,31 & 4,19 & 8,57 & 3,09 \\
3 & 73,33 & 15,78 & 4,14 & 8,54 & 3,06 \\
4 & 72,11 & 16,58 & 4,03 & 8,56 & 3,12 \\
\hline
\end{tabular}




\begin{tabular}{|c|c|c|c|c|c|}
\hline Dia/07/13 & Alizarol ${ }^{\circ} \mathbf{G L}$ & $\operatorname{Acidez}^{\circ} \mathbf{D}$ & Gordura \% & ESD \% & Proteína \% \\
\hline 5 & 73,56 & 16,33 & 3,97 & 8,50 & 3,09 \\
\hline 6 & 72,67 & 15,71 & 4,11 & 8,46 & 3,07 \\
\hline 7 & 73,20 & 17,00 & 4,17 & 8,59 & 3,10 \\
\hline 8 & 72,50 & 17,08 & 3,93 & 8,48 & 3,08 \\
\hline 9 & 73,00 & 15,58 & 4,14 & 8,45 & 3,06 \\
\hline 10 & 73,11 & 15,89 & 4,26 & 8,41 & 3,05 \\
\hline 11 & 73,00 & 16,38 & 4,13 & 8,42 & 3,06 \\
\hline 12 & 74,00 & 15,58 & 4,08 & 8,47 & 3,08 \\
\hline 13 & 74,00 & 15,17 & 4,33 & 8,42 & 3,06 \\
\hline 14 & 74,00 & 16,00 & 4,17 & 8,38 & 3,04 \\
\hline 15 & 75,50 & 15,38 & 4,07 & 8,36 & 3,04 \\
\hline 16 & 77,00 & 15,50 & 3,94 & 8,35 & 3,04 \\
\hline 17 & 75,20 & 15,30 & 6,39 & 8,52 & 3,08 \\
\hline 18 & 74,33 & 15,50 & 4,04 & 8,50 & 3,08 \\
\hline 19 & 72,67 & 16,63 & 4,10 & 8,53 & 3,08 \\
\hline 20 & 73,33 & 16,38 & 3,81 & 8,42 & 3,06 \\
\hline 21 & 75,56 & 15,47 & 4,02 & 8,42 & 3,05 \\
\hline 22 & 74,67 & 15,17 & 4,05 & 8,48 & 3,08 \\
\hline 23 & 73,25 & 16,67 & 4,03 & 8,46 & 3,06 \\
\hline 24 & 72,67 & 15,89 & 4,02 & 8,50 & 3,08 \\
\hline 25 & 74,33 & 16,17 & 4,06 & 8,54 & 3,10 \\
\hline 26 & 73,00 & 16,08 & 4,11 & 8,45 & 3,07 \\
\hline 27 & 73,33 & 16,33 & 4,19 & 8,60 & 3,13 \\
\hline 28 & 72,67 & 16,25 & 4,13 & 8,46 & 3,08 \\
\hline 29 & 75,17 & 15,33 & 4,14 & 8,45 & 3,07 \\
\hline 30 & 74,67 & 15,83 & 3,99 & 8,53 & 3,10 \\
\hline 31 & 74,60 & 15,92 & 3,91 & 8,44 & 3,07 \\
\hline Média & 73,38 & 15,96 & 4,15 & 8,47 & $\mathbf{3 , 0 7}$ \\
\hline
\end{tabular}

Fonte: Elaborado pelo Autor 


\subsubsection{Alizarol ${ }^{\circ} \mathbf{G L}$}

A Figura 1 nos apresenta a carta de controle dos índices de Alizarol presentes no leite.

FIGURA 1: ANÁLISE DE ALIZAROL DO LEITE CRU REFRIGERADO ENTREGUE NA INDÚSTRIA REFERENTE AO MÊS DE JULHO DE 2013

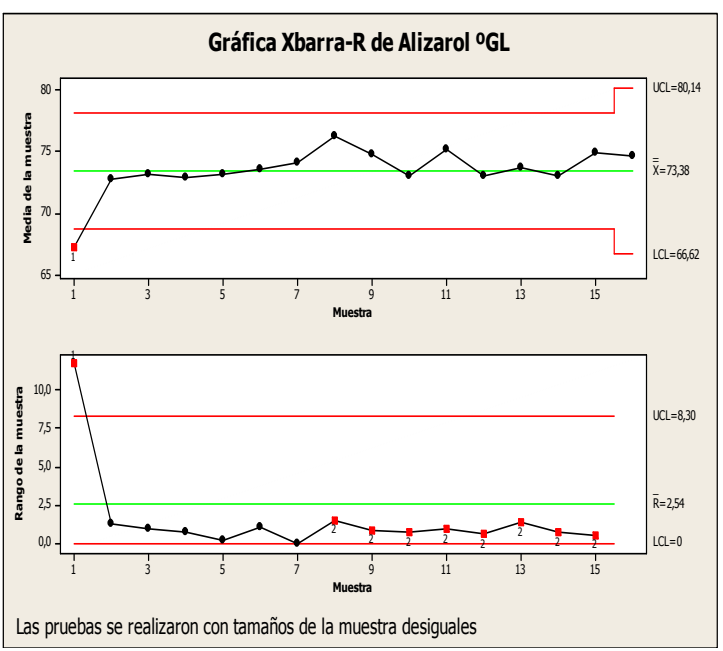

No gráfico da carta de controle $\mathrm{X}$, podemos notar que há um ponto fora do limite de controle, isto pode ter ocorrido devido a erro de medição ou anotação, a quebra do equipamento de medição ou o mesmo estar desregulado ou até mesmo a falta de treinamento do operador do equipamento.

Nota-se que os dados, excetuando o primeiro que se encontra fora dos limites de controle, respeitam os limites impostos pela IN 62 que é de no mínimo $72 \%$. No gráfico da carta de controle R, verificamos também que o mesmo ponto fora do controle da carta $\mathrm{X}$ é o ponto que sai do limite de controle da carta $\mathrm{R}$ e que uma sequência de 14 valores aparecem abaixo da linha central, o que pode indicar mudança no ajuste do aparelho de medição, método ou material diferente, avaria de um componente do leite, quebra do equipamento de medição ou grande variação no material recolhido. Sendo assim, notamos que esse processo está fora do controle estatístico da qualidade e que é preciso realizar uma ação sobre o processo antes que apareçam mais pontos fora dos limites de controle.

\subsubsection{Acidez}

A Figura 2 nos mostra o gráfico de cartas de controle para os índices de acidez.

FIGURA 2: ANÁLISE DO GRAU DE ACIDEZ DO LEITE CRU REFRIGERADO ENTREGUE NA INDÚSTRIA REFERENTE AO MÊS DE JULHO DE 2013

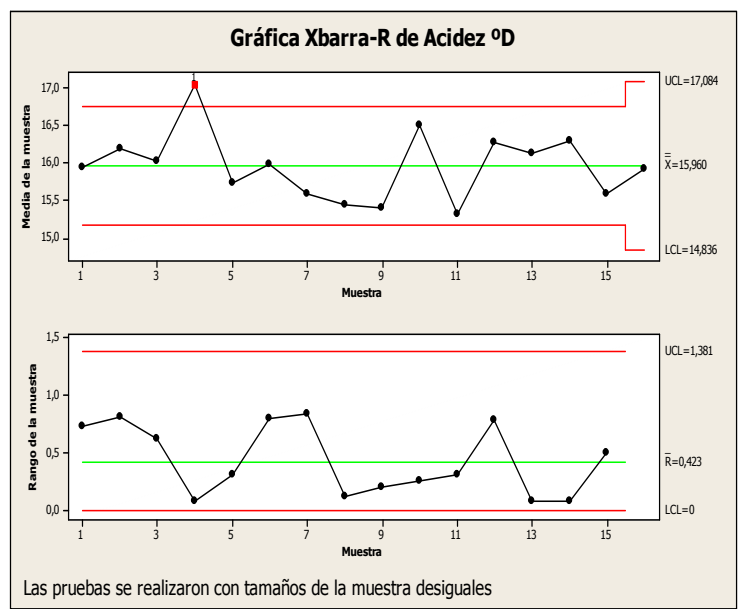

Na carta $X$, notamos que há um ponto fora dos limites de controle, o que também pode ser justificado por ter ocorrido um erro de medição ou anotação, a quebra do equipamento de medição ou o mesmo estar desregulado ou até mesmo a falta de treinamento do operador do equipamento. E é possível de notar que os dados estão em conformidade com a IN62, exceto a amostra 4 que apresenta fora dos limites de controle. 


\section{ReLAInEPY}

A carta $\mathrm{R}$, notamos que está tudo em conformidade, mas como a carta $\mathrm{X}$ teve valores fora dos limites, podemos concluir que o processo está fora do controle estatístico da qualidade.

\subsubsection{Teor de Gordura}

A Figura 3, apresenta os dados coletados e o valor estipulado pela IN 62 para o leite cru refrigerado mantenha em sua composição no mínimo $3 \%$ de gordura.

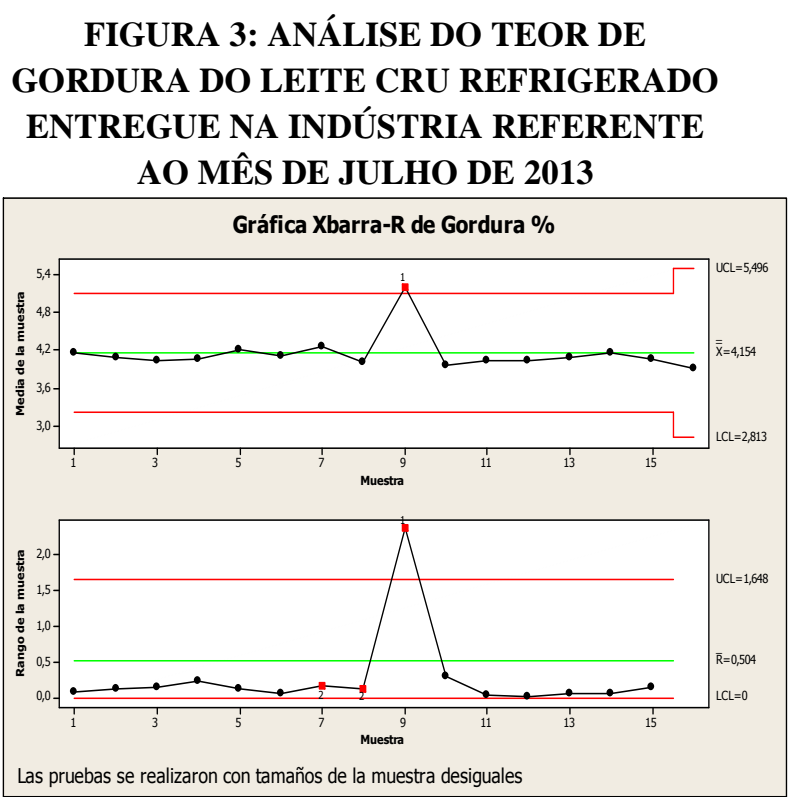

Na carta X, há pontos fora dos limites de controle e uma sequência de sete valores abaixo da linha central, o que pode indicar mudança no ajuste do aparelho de medição, método ou material diferente, avaria de um componente do leite, quebra do equipamento de medição ou grande variação no material recolhido. $\mathrm{O}$ mesmo pode ser observado na carta $R$, que também possui um valor fora dos limites de controle. Sendo assim, o processo está fora do controle estatístico da qualidade, mas estão em conformidade com a IN62 que tolera de 3 a 6
$\%$ o teor de gordura no leite. Mesmo assim, podemos concluir que o processo está sobre o controle estatístico da qualidade respeitando os limites de controle, mas como apresenta padrões não aleatórios na sequência de pontos é preciso realizar uma ação sobre o processo antes que um ponto apareça fora dos limites de controle.

\subsubsection{Teor de Proteína}

A Figura 4 nos apresenta a representação gráfica dos dados coletados da análise de proteína no leite.

FIGURA 4 - ANÁLISE DO TEOR DE PROTEÍNA DO LEITE CRU REFRIGERADO ENTREGUE NA INDÚSTRIA REFERENTE AO MÊS DE JULHO DE 2013

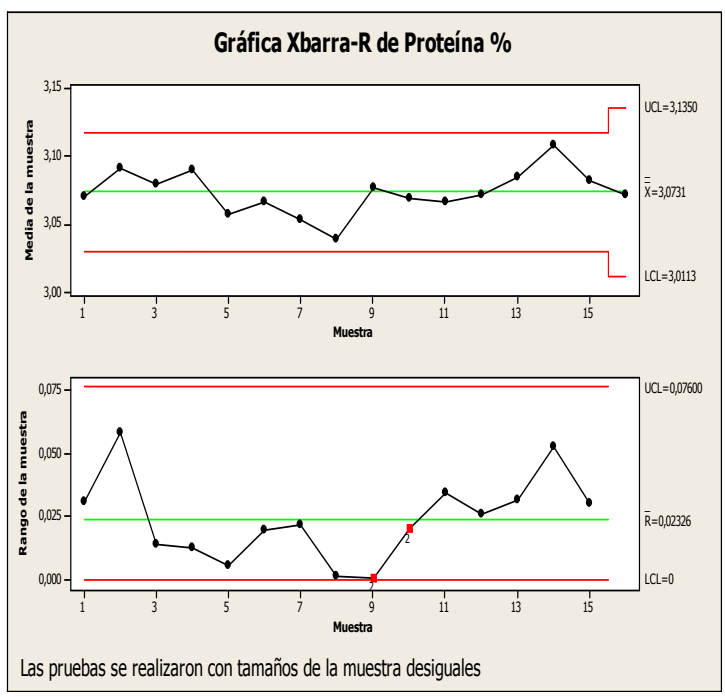

A carta $\mathrm{X}$ nos mostra que o processo está sobre o controle estatístico da qualidade, e as amostras respeitam os limites impostos pela IN62. Já na carta $R$, notamos que existe uma sequencia de sete pontos abaixo da linha média, o que implica em situações como aconteceu com os teores de gordura, onde poderia ter ocorrido falhas no processo como, mudança no ajuste 


\section{ReLAlnEP}

do aparelho de medição, método ou material diferente, avaria de um componente do leite, quebra do equipamento de medição ou grande variação no material recolhido. O que nos faz concluir que o processo está sobre controle estatístico da qualidade, mas como apresenta padrões não aleatórios na sequência de pontos é preciso realizar uma ação sobre o processo antes que um ponto apareça fora dos limites de controle.

\subsubsection{Extrato Seco Desengordurado (ESD)}

Os níveis de ESD estudados podem ser analisados na Figura 5.

FIGURA 5: ANÁLISE DO ESD DO LEITE CRU REFRIGERADO ENTREGUE NA INDÚSTRIA REFERENTE AO MÊS DE JULHO DE 2013

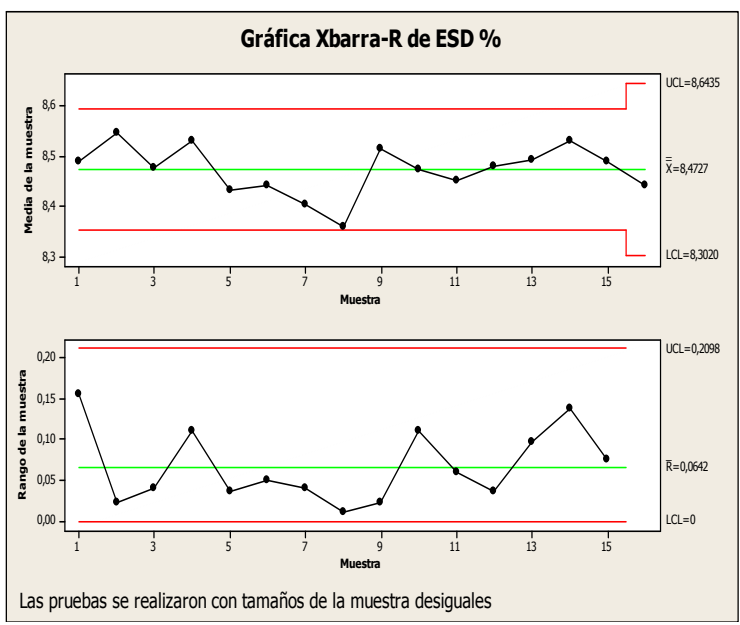

Tanto a carta X quanto a carta $\mathrm{R}$, estão sobre o controle estatístico do processo com todos os valores respeitando os limites de controle estatístico e os limites impostos pela IN62.

\subsection{Discussões dos resultados}

Diante do exposto, podemos notar que o processo não está totalmente sobre controle, já que apresenta pontos fora dos limites de controle do processo. As análises da lizarol apresentam índices fora de controle e abaixo do recomendado pela IN 62. A acidez do leite está fora de controle estatístico, já que possui índices que estão acima do limite de controle do processo e do tolerável pela normativa. Como a normativa impõe um teor de gordura de $3 \%$ ao leite em sua composição, não está sobre controle, já que há um ponto fora dos limites de controle. Podemos analisar que o teor de proteína e de ESD estão sobre controle estatístico do processo, com todos os índices dentro dos limites de controle e dentro do limite imposto pela normativa. Verificamos também, que os teores de proteína, gordura e alizarol apresentam um comportamento nao aleatório ou sistemático, o que pode contribuir para indício de causas especiais no processo, portanto é preciso realizar uma ação sobre o processo antes que mais pontos apareçam fora dos limites de controle.

\section{Conclusões}

Assim, concluímos que segundo as análises de proteína e de estrato de sólido desengordurado estão sobre controle e dentro do limite imposto pela IN 62. Já as análises de Alizarol, teor de gordura e acidez, apresentaram índices fora dos limites de controle e fora dos limites impostos pela normativa. Os teores de proteína, 
gordura e Alizarol apresentam um comportamento não aleatório ou sistemático, o que pode contribuir para indício de causas especiais no processo, portanto é preciso realizar uma ação sobre o processo antes que mais pontos apareçam fora dos limites de controle.

Diante disso, é preciso uma atenção maior aos índices que se apresentaram fora de controle, podendo empregar outras ferramentas da qualidade como brainstorming, diagrama de Ishikawa, estratificação, com o objetivo de levantar as causas das inconformidades. Isso levaria a uma investigação minuciosa de toda cadeia de produção do leite, desde o manejo com os animais, condições de higiene das instalações das salas de ordenha até condições de transporte, equipamentos de medição e manuseio da matéria-prima.

\section{Referências}

ALVIM, Rodrigo Sant'Anna. Panorama do setor lácteo brasileiro. 2013. Disponível em: <http://www.senado.gov.br/comissoes/CRA/ AP/AP20130404_RodrigoAlvim.pdf $>$. Acesso em 17 de setembro de 2013.

BONDUELLE, Ghislaine. Ferramentas de Controle. Disponível em: <http://www.madeira.ufpr.br/portal12/down loads/ghislaine/cep.pdf $>$. Acesso em 23 de setembro de 2013.

BRITO, M.A.; BRITO, J.R. Qualidade do leite. Disponível em: $<$ http://www.fernandomadalena.com/site_arq uivos/903.pdf $>$. Acesso em 17 de setembro de 2013.

DÜRR, João Walter. Como produzir leite de qualidade. 4. ed. Brasília: SENAR, 2012. 44 p. il. ; $21 \mathrm{~cm}$. (Coleção SENAR, ISSN 1676-367x, 113).

FERREIRA, M. A. Controle de qualidade físico-químico em leite fluído. Universidade de
Brasília (UnB). Disponível em: $<$ http://www.emater.pr.gov.br/>. Acesso em 17 de setembro de 2013.

FRANCO, Bruno Souza; et al. Análises das enzimas peroxidase e fosfatase em amostras de leite cru, pasteurizado e londa vida. 2011. Disponível em: <http://www.hestia.org.br/novo/revista01/d oc/artigos/CITINO_37_No1_Ed1_Ano1_Port. pdf $>$. Acesso em 17 de setembro de 2013.

LEÃO, Jéssica Samara; ALVES, Jociane Maria; SILVA, Fellipe Borges; COSTA, Daviane Martinelle; NASCIMENTO, Gian Carlos; CEACERO, Thais Matos; TEIXEIRA, Rafael Bastos; CARVALHO, Rafael Mendonça de. Análises físicoquímicas do leite produzido nas propriedades assistidas pelo Programa "MAIS LEITE”. $2012 . \quad$ Disponível em: <http://www.cefetbambui.edu.br/portal/files / An\%C3\%A1lises\%20f\%C3\%ADsicoqu $\%$ C $3 \%$ ADmicas $\% 20$ do $\% 2$

Oleite $\% 20$ produzido $\% 20$ nas $\% 20$ propriedades $\% 2$ Oassistidas\%20pelo\%20Programa\%20_MAIS\%2 OLEITE_.pdf $>$. Acesso em 25 de setembro de 2013.

LEITE. ANEXO IV REGULAMENTO TÉCNICO DE IDENTIDADE E QUALIDADE DE LEITE CRU REFRIGERADO. Disponível em: <http://www.leite.pr.gov.br/arquivos/File/le gislacao/anexo_IV.pdf $>$. Acesso em 23 de setembro de 2013.

MAPA, Ministério da Agricultura e do Meio Ambiente. INSTRUÇÃO NORMATIVA No 51 DE 18/09/2002. Disponível em: <http://adcon.rn.gov.br/ACERVO/EMATER /DOC/DOC000000000001051.PDF>. Acesso em 23 de setembro de 2013.

MELO, Karine Coelho. Utilização das cartas de controle de média para avaliação do peso em sorvetes. 2004. Disponível em: <http: / / professor.ucg.br/SiteDocente/admin/ arquivosUpload/8930/material/TCC-

Kari-

ne $\% 20$ UTILIZA $\%$ C3\%87\%C3\%83O\%20DAS\% 20CARTAS\%20DE\%20CONTROLE\%20DE\%2 OM\%C3\%89DIA \%20PARA\%20AVALIA\%C3\% $87 \% \mathrm{C} 3 \% 83 \mathrm{O} \% 20 \mathrm{DE} \% 20 \mathrm{PESO} \% 20 \mathrm{EM} \% 20 \mathrm{SO}$ RVETES.pdf $>$. Acesso em 25 de setembro de 2013.

MIGUEL, Giuliana Zilocchi; MAGALHÃES, Marcelo Correia de; GERON, Luiz Juliano Valério; BOTINI, Tatiani; SAENZ, Edgar Collao; CRUZ, 
Cristiano da. Caracterização físico-química de leite obtido de diferentes tipos de comercialização em Pontes e Lacerda - MT. Disponível

em: $<$ http://www.unemat.br/revistas/rcaa/docs/v ol8/10_artigo_v8.pdf $>$. Acesso em 23 de setembro de 2013.

RODRIGUES, Fernando. Manual de bancada boas práticas de laboratório. 2005. Disponível em:

<http://www.queijosnobrasil.com.br/downloa ds/manualbancada.pdf $>$. Acesso em 25 de setembro de 2013.

SILVA, Paulo Henrique Fonseca da. Leite: Aspectos de composição e propriedades. 1997. Disponível em: <http://qnesc.sbq.org.br/online/qnesc06/qui msoc.pdf $>$. Acesso em 17 de setembro de 2013. 